\title{
SCIENTIFIC REPORTS

\section{Collagen fragments quantified in serum as measures of desmoplasia associate with survival outcome in patients with advanced pancreatic cancer}

\author{
Nicholas Willumsen ${ }^{1 *}$, Suhail M. Ali ${ }^{2,3}$, Kim Leitzel ${ }^{2}$, Joseph J. Drabick ${ }^{2}$, Nelson Yee ${ }^{2}$, \\ Hyma V. Polimera ${ }^{2}$, Vinod Nagabhairu ${ }^{4}$, Laura Krecko ${ }^{2}$, Ayesha Ali ${ }^{2}$, Ashok Maddukuri ${ }^{2}$, \\ Prashanth Moku', Aamnah Ali², Joyson Poulose ${ }^{2}$, Harry Menon², Neha Pancholy ${ }^{2}$, \\ Luis Costa ${ }^{5,6}$, Morten A. Karsdal ${ }^{1}$ \& Allan Lipton ${ }^{2}$
}

Pancreatic ductal adenocarcinoma (PDAC) patients have poor prognosis and poor response to treatment. This is largely due to PDAC being associated with a dense and active stroma and tumor fibrosis (desmoplasia). Desmoplasia is characterized by excessive degradation and formation of the extracellular matrix (ECM) generating collagen fragments that are released into circulation. We evaluated the association of specific collagen fragments measured in pre-treatment serum with outcome in patients with PDAC. Matrix metalloprotease (MMP)-degraded type I collagen (C1M), type III collagen (C3M), type IV collagen (C4M) and a pro-peptide of type III collagen (PRO-C3) were measured by ELISA in pre-treatment serum from a randomized phase 3 clinical trial of patients with stage III/ IV PDAC treated with 5 -fluorouracil based therapy $(n=176)$. The collagen fragments were evaluated for their correlation ( $r$, Spearman) with serum CA19-9 and for their association with overall survival (OS) based on Cox-regression analyses. In this phase 3 PDAC trial, pre-treatment serum collagen fragment levels were above the reference range for $67 \%-98 \%$ of patients, with median values in PDAC approximately two-fold higher than reference levels. Collagen fragment levels did not correlate with CA19-9 $(r=0.049-0.141, p=n s)$. On a continuous basis, higher levels of all collagen fragments were associated with significantly shorter OS. When evaluating degradation (C3M) and formation (PRO-C3) of type III collagen further, higher PRO-C3 was associated with poor OS $>25^{\text {th }}$ percentile cut-point, $\mathrm{HR}=2.01,95 \% \mathrm{Cl}=1.33-3.05)$ and higher $\mathrm{C} 3 \mathrm{M} / \mathrm{PRO}-\mathrm{C} 3$ ratio was associated with improved OS ( $>25^{\text {th }}$ percentile cut-point, $\mathrm{HR}=0.53,95 \% \mathrm{Cl}=0.34-0.80$ ). When adjusting for CA19-9 and clinical covariates, PRO-C3 remained significant $(\mathrm{HR}=1.65,95 \% \mathrm{Cl}=1.09-2.48)$. In conclusion, collagen remodeling quantified in pre-treatment serum as a surrogate measure of desmoplasia was significantly associated with OS in a phase 3 clinical PDAC trial, supporting the link between desmoplasia, tumorigenesis, and response to treatment. If validated, these biomarkers may have prognostic and/or predictive potential in future PDAC trials.

Pancreatic cancer (PC) is one of the most lethal types of cancer with an overall 5-year survival rate of $8 \%$ dropping to $3 \%$ in the metastatic setting ${ }^{1}$. At time of diagnosis, approximately $10 \%$ of PC patients have tumors that are potentially curable with resection, and 50\% have metastatic disease. In 2017, in the United States alone, 55,440 new cases and 44,330 deaths were recorded. It has been projected that PC will become the second leading cause of

${ }^{1}$ Biomarkers \& Research, Nordic Bioscience, Herlev, Denmark. ${ }^{2}$ Division of Hematology/Oncology, Penn State Health Milton S Hershey Medical Center, Hershey, PA, USA. ${ }^{3}$ Lebanon VA Medical Center, Lebanon, PA, USA. ${ }^{4}$ Pinnacle Health System, University of Pittsburgh Medical Center, Harrisburg, PA, USA. ${ }^{5}$ Oncology division, Hospital de Santa Maria, Lisboa, Portugal. ${ }^{6} \mathrm{Clinical}$ Translational Oncology Research Unit, Institute of Molecular Medicine, Lisboa, Portugal.*email: nwi@nordicbio.com 
cancer-related death in the United States in the next decade, hence PC is a clear exception from the general trend of improvement in survival rates for patients with other cancers ${ }^{1,2}$.

Not only is PC diagnosed late, but patients with advanced PC also respond poorly to chemo- and targeted-therapies ${ }^{3,4}$. Currently, besides imaging (computed tomography), carbohydrate antigen 19-9 (CA19-9), an antigen released by pancreatic cancer cells and measured in serum, is the only recommended biomarker for predicting and monitoring outcome in the PC setting 5 . Taken together, the grim PDAC outcome emphasizes the need not only for innovative drug development efforts, but also for the development of biomarkers that can be used for stratifying PC patients and predicting their outcome and likelihood of response to treatment.

Pancreatic ducal adenocarcinoma (PDAC) is the most common form of PC and accounts for more than $80 \%$ of cases. PDAC is the most stromal cancer type across all tumor indications, characterized by an extremely dense and desmoplastic (fibrotic) tumor microenvironment ${ }^{6,7}$. The desmoplastic reaction is highly associated with therapy resistance and tumorigenesis, thus accounting for the lack of satisfactory outcomes for PDAC patients ${ }^{8,9}$.

Desmoplasia is characterized by an altered remodeling of the collagenous extracellular matrix (ECM), which is the non-cellular component of tissue. While the ECM turnover in healthy tissue is maintained in a delicate homeostatic state between ECM/collagen formation and degradation, this homeostasis is lost in the tumor tissue $^{10-13}$. Upregulation of the production and assembly of collagens, i.e. the formation of a fibrous connective tissue, is driven by proliferation and activation of myofibroblasts and myofibroblast-like stellate cells ${ }^{14,15}$. An associated collagen degradation is concomitantly observed, and is driven by a highly proteolytically-active tumor microenvironment, in which the matrix metalloproteases (MMPs) play a key role in degrading collagens ${ }^{16,17}$.

The interstitial matrix-associated type I collagen, type III collagen, and the basement membrane-associated type IV collagen have all been found upregulated in the primary tumors and metastatic lesions of PDAC, and are associated with poor outcome and pro-tumorigenic events ${ }^{7,18-21}$. Type IV collagen is a network forming collagen that supports the epithelia/endothelia whereas type I and type III collagen are fibrillar collagens part of the underlying stromal interstitial matrix. While tumor cell invasion and angiogenesis are associated with remodeling and degradation of type IV collagen, the synthesis, deposition, remodeling and degradation of the fibrillar collagens is more directly linked to fibroblast activity ${ }^{21-25}$.

A pilot study has evaluted the biomarker potential of specific MMP-derived fragments of type I (C1M), type III (C3M), and type IV (C4M) collagen as surrogate measures of desmoplasia and ECM remodeling, and found that all these fragments were elevated in serum from patients with PDAC compared to healthy controls ${ }^{26}$. Based on these findings, and the importance of desmoplasia in PDAC, we hypothesized that $\mathrm{C} 1 \mathrm{M}, \mathrm{C} 3 \mathrm{M}, \mathrm{C} 4 \mathrm{M}$, as well as PRO-C3, a biomarker derived from the pro-peptide of type III collagen, were predictive of outcome in the PDAC setting.

The aim of this study was to evalute how levels of specific collagen fragments (C1M, C3M, C4M, PRO-C3), quantified in pre-treatment serum, were associated with overall survival (OS) in a randomized phase 3 clinical trial of patients with advanced unresectable PDAC. The association between the collagen markers and CA19-9, the gold-standard PDAC serum biomarker, and outcome was also evaluated.

\section{Methods}

Patients and study design. The present study includes 176 patients with advanced unresectable PDAC that were enrolled in a randomized, double-blinded, placebo-controlled, multicenter phase III trial. One arm received octreotide (somatostatin, a somatotrophin-release inhibiting factor analogue) and continuous infusion of 5-fluorouracil (5-FU) and another arm received placebo and 5-FU ${ }^{27}$.

Briefly, patient inclusion criteria included (1) at least 18 years of age; (2) measurable or evaluable, stage III or IV, pathologically, histologically, or cytologically confirmed unresectable adenocarcinoma of the exocrine pancreas; and (3) no prior chemotherapy, radiation, or hormonal therapy ${ }^{28}$. The trial was powered to achieve 330 evaluable patients (165 per arm) with planned randomization of 412 patients (206 per arm) to detect an increase in 1-year survival rate from $10 \%$ in the 5 -FU + placebo group to $20 \%$ in the 5 -FU + octreotide group with a power of 0.85 . The primary objective of this trial was overall survival (OS); secondary objectives were progression-free survival (PFS), clinical benefit, tolerability of octreotide, and objective response rate. Treatment after randomization was octreotide ( $160 \mathrm{mg}$ intramuscular initiation, every 2 weeks for four injections and then every 4 weeks until disease progression) vs. placebo; 5 -FU was given immediately following the first octreotide injection, by continuous intravenous infusion at a total daily dose of $225 \mathrm{mg} / \mathrm{m} 2$, for 8 weeks, followed by a 7-day rest period, and then, 5-FU was repeated until disease progression. The trial was stratified for gender, Karnofsky performance status (KPS, $70-100 \%$ vs. 50-60\%), and stage of disease (III vs. IV).

The interim efficacy analysis of this clinical trial reported that the median OS time in the octreotide arm was 22.6 weeks (95\%CI: 18.1-27.7) vs. 21.6 weeks in the placebo arm (95\%CI: 17.9-28.3); this was not a significant difference $(\mathrm{P}=0.649)$ in OS between the two arms $(27)$. There was one complete (stage III, in octreotide arm) and two partial remissions (both in stage IV; one in octreotide arm and one in placebo arm). Since there was no efficacy difference in the two arms of this trial, it is optimal for biomarker discovery and validation. This same cohort had previously been used to report a significant association between higher inflammatory serum biomarkers (ferritin and C-reative protein, CRP) and shorter $\mathrm{OS}^{28}$.

Serum biomarker measurements. Biomarker levels were measured in pre-treatment serum samples. C1M (MMP-degraded type I collagen, originally described by Leeming et al. ${ }^{29}$ ), C3M (MMP-degraded type 3 collagen, originally described by Veidal et al..$^{30}$ ), C4M (MMP-degraded type IV collagen, originally described by Veidal et al..$^{31}$ ) and PRO-C3 (N-terminal pro-peptide of type III collagen, originally described by Nielsen et al. ${ }^{32}$ ) were quantified by individual competitive enzyme-linked immune sorbent associated assays (ELISAs) according to the manufacturer's instructions (Nordic Bioscience, Herlev, Denmark). The normal reference range (mean with 


\begin{tabular}{|l|l|l|l|l|}
\hline & C1M & C3M & C4M & PRO-C3 \\
\hline Minimum, ng/ml & 20.0 & 6.0 & 8.8 & 5.0 \\
\hline 25\% Percentile, ng/ml & 23.4 & 15.1 & 35.7 & 10.4 \\
\hline Median, ng/ml & 44.7 & 20.5 & 46.9 & 17.7 \\
\hline $75 \%$ Percentile, ng/ml & 84.4 & 26.3 & 64.0 & 33.1 \\
\hline Maximum, ng/ml & 362.9 & 58.7 & 123.0 & 201.8 \\
\hline \% above reference range & $67 \%$ & $93 \%$ & $98 \%$ & $86 \%$ \\
\hline
\end{tabular}

Table 1. Overview of collagen fragment biomarker levels in pre-treatment serum from patients with pancreatic ductal adenocarcinoma (PDAC).

95\% CI) for the collagen markers were pre-defined from a population of 617 healthy men and women ranging from 22 to 86 years of age ${ }^{33}$. The upper limit of the reference range was $28.4 \mathrm{ng} / \mathrm{ml}$ for C1M, $10.3 \mathrm{ng} / \mathrm{ml}$ for C3M, $19.3 \mathrm{ng} / \mathrm{ml}$ for C4M and $8.7 \mathrm{ng} / \mathrm{ml}$ for PRO-C3. Biomarker values below/above the detection limit were assigned the lower/upper limit of detection value for each respective assay.

Statistical analysis. The serum collagen fragments (C1M, C3M, C4M and PRO-C3) were analyzed for an association with OS by use of univariate and multivariate Cox proportional hazards modeling. Biomarkers (variables) were evaluated both on a continuous and categorical scale, the latter by dichotomizing the variables by separation at the $25^{\text {th }}$ percentile cut-point. The dichotomized variables were also evaluated by Kaplan-Meier survival estimates (OS curves). Correlations between serum collagen fragments and CA19-9 were evaluated by calculating the Spearman rank correlation coefficients. A p-value $<0.05$ was considered statistically significant.

Ethics approval and consent to participate. Signed informed consent to participate in the present study was obtained from all patients before sample collection. This study was reviewed and approved by the institutional review boards at the Pennsylvania State University Hershey Medical Center and performed in accordance with the Declaration of Helsinki ${ }^{28}$.

\section{Results}

Patient demographics. The demographics and patient characteristics are shown in Supplementary Tables 1 and 2 .

Levels of collagen fragments in serum from patients with PDAC. An overview of the distribution of the collagen fragments $\mathrm{C} 1 \mathrm{M}, \mathrm{C} 3 \mathrm{M}, \mathrm{C} 4 \mathrm{M}$ and $\mathrm{PRO}-\mathrm{C} 3$ in pre-treatment serum samples from 176 patients with advanced PDAC is shown in Table 1 and Fig. 1. Overall, most of patients presented with biomarker levels above the reference range and with median values in PDAC approximately two-fold higher than the reference range.

Correlations between collagen fragments and CA19-9. Table 2 shows the Spearman rank correlation coefficients $(\mathrm{r})$ between the collagen markers (C1M, C3M, C4M and PRO-C3) and CA19-9. While all collagen fragments correlated with each other $(\mathrm{r}=0.462-0.897, p<0.0001)$, no correlation was seen between any of the collagen markers and CA19-9 $(\mathrm{r}=0.049-0.141, p=0.065-0.521)$. This indicates that the collagen fragments represent similar pathological events, and that these are distinct from the pathological events linked to CA19-9.

Association between collagen fragments in serum and clinical demographics and overall survival (OS) outcome. First, when evaluating the association between stage of disease and Karnofsky performance status (KPS) and collagen fragments, serum C1M and PRO-C3 were significantly higher in patients with Stage IV vs Stage II and III disease (Supplementary Table 2). Similarly, serum C1M, C3M, and PRO-C3 were significantly higher in patients with a KPS of 1 vs 0.

When evaluated by univariate analysis (Table 3), C3M, C4M and PRO-C3 predicted for significantly shorter OS when evaluated on a continuous scale $(p=0.004-0.037)$, while $\mathrm{C} 1 \mathrm{M}$ was only borderline significant $(p=0.056)$. In contrast, the C3M/PRO-C3 ratio predicted for improved OS $(p=0.035)$. When evaluating the four collagen fragments individually on a categorical scale by dichotomization at the $25^{\text {th }}$ percentile cut-point, patients above the $25^{\text {th }}$ percentile cut-point had shorter OS. Patients had an approximately 1.5 -fold increased risk of dying if presenting with high levels of $\mathrm{C} 1 \mathrm{M}(\mathrm{HR}=1.54, p=0.025), \mathrm{C} 3 \mathrm{M}(\mathrm{HR}=1.44, p=0.053)$ and $\mathrm{C} 4 \mathrm{M}(\mathrm{HR}=1.47$, $p=0.039)$, respectively. Patients with high levels of $\mathrm{PRO}-\mathrm{C} 3 \mathrm{had}$ a 2 -fold increased risk of dying $(\mathrm{HR}=2.01$, $p=0.001)$. Again, high $\mathrm{C} 3 \mathrm{M} / \mathrm{PRO}-\mathrm{C} 3$ predicted for a survival benefit with ratio-high patients having a $47 \%$ reduced risk of dying $(\mathrm{HR}=0.53, p=0.002)$.

Next, an association between the collagen fragments and OS was evaluated by Kaplan-Meier plots using the $25^{\text {th }}$ percentile cut-point (Fig. 2). Overall, high levels of serum collagen fragments were associated with shorter OS. Median OS for patients with high vs low ( $>25 \%$ vs $\leq 25 \%$ ) C1M was 133 days vs. 223 days; high vs low C3M was 139 days vs. 174 days; high vs low C4M was 139 days vs. 174 days, and high vs low PRO-C3 was 127 days vs. 264 days. When evaluating the $\mathrm{C} 3 \mathrm{M} / \mathrm{PRO}-\mathrm{C} 3$ ratio, median OS for patients with a high C3M/PRO-C3 ratio was 172 days vs 109 days for patients with a low C3M/PRO-C3 ratio (not shown). 

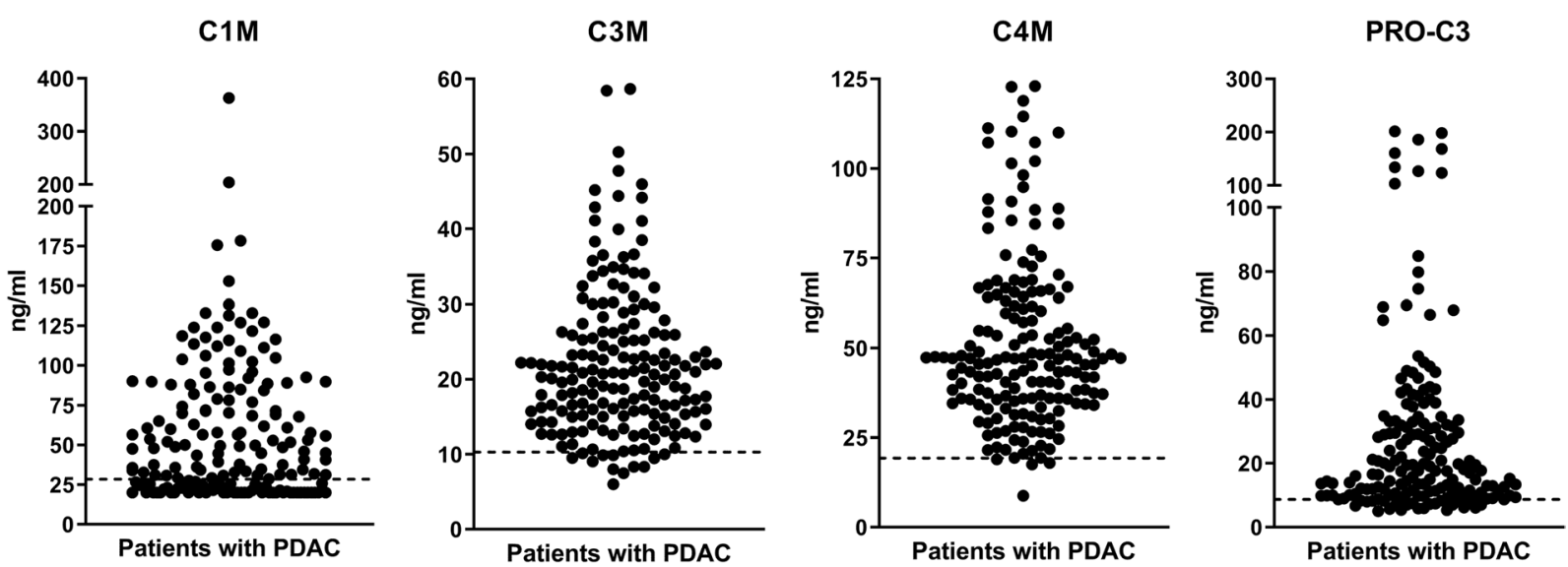

Figure 1. Levels of collagen fragments (C1M, C3M, C4M, PRO-C3) in pre-treatment serum from 176 patients with advanced pancreatic ductal adenocarcinoma (PDAC). C1M measures MMP-degraded type I collagen, C3M measures MMP-degraded type 3 collagen, C4M measures MMP-degraded type IV collagen, and PROC3 measures the N-terminal pro-peptide of type III collagen. The dotted line represents the upper limit of the normal reference range (defined as mean with 95\%CI) from a population of 617 healthy men and women ranging from 22 to 86 years of age $e^{33}$.

\begin{tabular}{|l|l|l|l|l|l|}
\hline & & C3M, ng/ml & C4M, ng/ml & PRO-C3, ng/ml & CA 19-9, U/ml \\
\hline $\begin{array}{l}\text { C1M, } \\
\text { ng/ml }\end{array}$ & $\begin{array}{l}\mathrm{r} \\
p \text {-value }\end{array}$ & $0.679<0.0001$ & $0.636<0.0001$ & $0.485<0.0001$ & 0.0840 .2751 \\
\hline $\begin{array}{l}\mathrm{C} 3 \mathrm{M}, \\
\mathrm{ng} / \mathrm{ml}\end{array}$ & $\begin{array}{l}\mathrm{r} \\
p \text {-value }\end{array}$ & - & $0.897<0.0001$ & $0.462<0.0001$ & 0.0490 .5213 \\
\hline $\begin{array}{l}\mathrm{C} 4 \mathrm{M}, \\
\mathrm{ng} / \mathrm{ml}\end{array}$ & $\begin{array}{l}\mathrm{r} \\
p \text {-value }\end{array}$ & & - & $0.516<0.0001$ & 0.1150 .1297 \\
\hline $\begin{array}{l}\text { PRO-C3, } \\
\text { ng/ml }\end{array}$ & $\begin{array}{l}\mathrm{r} \\
p \text {-value }\end{array}$ & & - & - & 0.1410 .0649 \\
\hline
\end{tabular}

Table 2. Spearman rank correlation coefficients (r) between the collagen markers (C1M, C3M, C4M and PROC3) and CA19-9.

\begin{tabular}{|c|c|c|c|}
\hline Biomarker & HR & $95 \%$ CI & $p$-value \\
\hline \multicolumn{4}{|c|}{ Continuous scale (n, events/total) } \\
\hline $\mathrm{C} 1 \mathrm{M}(16 / 149)$ & 1.002 & $0.999-1.005$ & 0.056 \\
\hline C3M (17/152) & 1.019 & $1.004-1.036$ & 0.015 \\
\hline C4M (17/152) & 1.007 & $1.0004-1.013$ & 0.037 \\
\hline PRO-C3 (16/149) & 1.001 & $1.006-1.014$ & 0.004 \\
\hline $\begin{array}{l}\text { C3M/PRO-C3 (ratio) } \\
(16 / 149)\end{array}$ & 0.770 & $0.610-0.982$ & 0.035 \\
\hline \multicolumn{4}{|c|}{ Categorical scale, $\leq 25^{\text {th }} v \mathrm{vs}>25^{\text {th }}$ percentile } \\
\hline $\mathrm{C} 1 \mathrm{M}$ & 1.54 & $1.06-2.25$ & 0.025 \\
\hline $\mathrm{C} 3 \mathrm{M}$ & 1.44 & $0.96-2.1$ & 0.053 \\
\hline $\mathrm{C} 4 \mathrm{M}$ & 1.47 & $1.02-2.15$ & 0.039 \\
\hline PRO-C3 & 2.01 & $1.33-3.05$ & 0.001 \\
\hline C3M/PRO-C3 & 0.53 & $0.35-0.80$ & 0.002 \\
\hline
\end{tabular}

Table 3. Univariate Cox proportional hazards modelling for evaluating the association between serum collagen fragments (C1M, C3M, C4M and PRO-C3) and overall survival (OS) in patients with advanced unresectable PDAC.

Lastly, multivariate analysis using the Cox proportional hazards model was used to evaluate the predictive value of the serum collagen fragments and clinical demographics with OS (Table 4). The serum collagen fragments were analyzed by dichotomization at the $25^{\text {th }}$ percentile cut-point, and clinical demographics included age (continuous), stage (categorical) and Karnofsky performance status (KPS) (categorical). In this multivariate analysis (Table 4) (adjusted for serum CA19-9), only serum PRO-C3 (HR 1.65, p=0.018), and stage (HR 1.33, $\mathrm{p}=0.03$ ) remained significant. Age, KPS, and the remaining serum collagen fragments $(\mathrm{C} 1 \mathrm{M}, \mathrm{C} 3 \mathrm{M}$, and $\mathrm{C} 4 \mathrm{M})$ were not significant. 

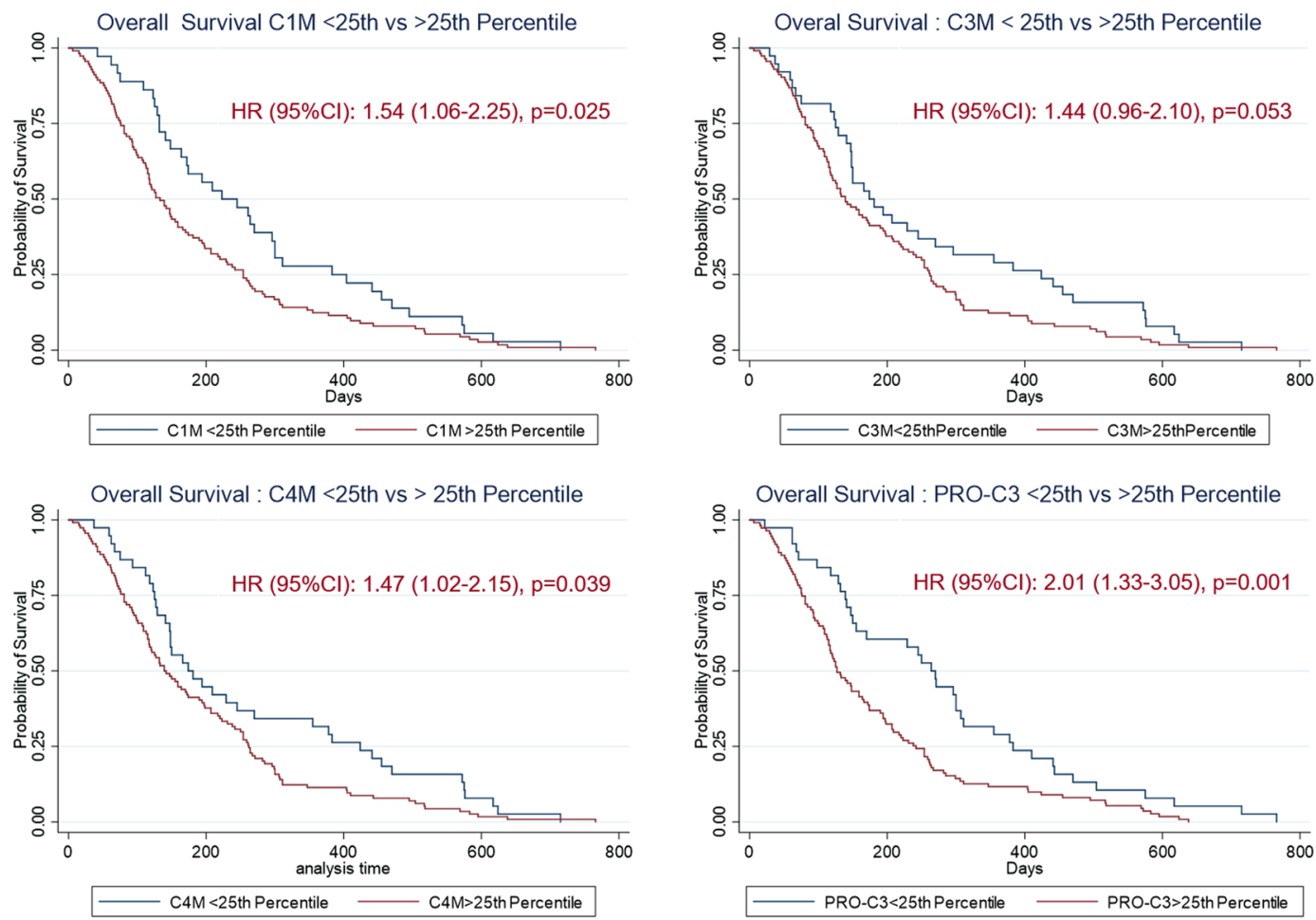

Figure 2. Probability of overall survival (OS) evaluated by Kaplan-Meier plots of patients with PDAC after dichotomizing the individual collagen biomarkers (C1M, C3M, C4M, PRO-C3) at the $25^{\text {th }}$ percentile cut-point. Hazard Ratio (HR) with $95 \%$ confidence interval $(95 \% \mathrm{CI})$ for patients with biomarker levels $>25^{\text {th }}$ percentile shown on each graph.

\begin{tabular}{|l|l|l|l|}
\hline Biomarker & HR & 95\% CI & p-value \\
\hline Age (continuous) & 1.003 & $0.986-1.021$ & 0.704 \\
\hline Stage (II + III vs IV) & 1.33 & $1.03-1.71$ & 0.030 \\
\hline KPS $(0$ vs 1$)$ & 0.79 & $0.44-1.40$ & 0.418 \\
\hline C1M, $25^{\text {th }}$ percentile & 1.03 & $0.64-1.67$ & 0.893 \\
\hline $\mathrm{C} 3 \mathrm{M}, 25^{\text {th }}$ percentile & 1.08 & $0.47-2.49$ & 0.856 \\
\hline $\mathrm{C} 4 \mathrm{M}, 25^{\text {th }}$ percentile & 1.27 & $0.57-2.86$ & 0.558 \\
\hline PRO-C3, $25^{\text {th }}$ percentile & 1.65 & $1.09-2.48$ & 0.018 \\
\hline
\end{tabular}

Table 4. Multivariate Cox proportional hazards modelling adjusted for serum CA19-9 for evaluating the predictive value of serum collagen fragments $(\mathrm{C} 1 \mathrm{M}, \mathrm{C} 3 \mathrm{M}, \mathrm{C} 4 \mathrm{M}$ and $\mathrm{PRO}-\mathrm{C} 3)$ and clinical demographics with overall survival (OS) in patients with advanced unresectable PDAC.

\section{Discussion}

PDAC is the most stroma-rich cancer type characterized by severe desmoplasia and ongoing ECM remodeling, both in the primary tumor and metastatic lesions ${ }^{7}$. Alterations in the tumor ECM not only associates with aggressive disease and impedes access/activity of anti-tumor therapy, but also generates circulating collagen fragments with pathologic fingerprints. In this study we measured such fingerprints in association with OS outcome.

The present results show that specific collagen peptide fingerprints that reflect collagen synthesis (PRO-C3) and collagen degradation $(\mathrm{C} 1 \mathrm{M}, \mathrm{C} 3 \mathrm{M}, \mathrm{C} 4 \mathrm{M})$ are highly elevated in patients with advanced unresectable PDAC compared to the healthy reference range, have a relatively broad dynamic range and associate with outcome when measured in serum prior to treatment with 5-FU-based therapy. These findings support what has been reported with the same markers in diagnostic (case-control) studies of lung cancer, breast cancer and ovarian cancer as well as prognostic studies of both metastatic breast cancer patients treated with letrozole or trastuzumab, and metastatic melanoma patients treated with ipilimumab ${ }^{34-37}$. Importantly, this study also confirms previous findings showing that MMP-mediated collagen turnover measured in serum by C1M, C3M and C4M is elevated in PDAC compared to healthy controls, as well as data showing prognostic value of $\mathrm{PRO}-\mathrm{C} 3$ measured in PC patients ${ }^{26,38}$.

Of the four collagen markers tested, $\mathrm{PRO}-\mathrm{C} 3$ and the $\mathrm{C} 3 \mathrm{M} / \mathrm{PRO}-\mathrm{C} 3$ ratio performed best in predicting OS outcome in PDAC. PRO-C3 measures the pro-peptide that is released when type III collagen assembles in the tissue. Type III collagen is synthesized by activated fibroblasts and is a major component of the desmoplastic reaction and ECM accumulation in the tumor. C3M is a measure of type III collagen degradation, therefore the 
$\mathrm{C} 3 \mathrm{M} / \mathrm{PRO}-\mathrm{C} 3$ ratio can be interpreted to reflect a reduced net fibrotic activity: high collagen degradation (C3M) with low collagen accumulation (PRO-C3). Clearly, this supports the notion that by measuring the surrogates of the desmoplastic reaction and altered turnover of the interstitial collagens (e.g. type III collagen), it is possible to identify patients with worsened prognosis ${ }^{39}$. In addition, PRO-C3 did not associate with CA19-9, indicating that PRO-C3 reflects a different underlying biology than the current gold standard PDAC serum biomarker (CA199), and hence may add additional value when evaluated to a greater extent in future clinical trials as they are produced by different cell types in the tumor microenvironment, i.e. cancer cells (CA19-9) and cancer associated fibroblasts (PRO-C3).

Previous findings have shown that collagen types I, III, and IV are concomitantly overexpressed together with hyaluronan (HA, a high-molecular-mass polysaccharide found in the ECM), predicting for poor prognosis and poor response to chemotherapy in $\mathrm{PC}^{7,40}$. This ultimately led to development of a recombinant pegylated hyaluronidase enzyme (PEGPH20, targeting HA), which would potentially increase drug uptake of chemotherapy ${ }^{41}$. A randomized phase II study (HALO-202) of PEGH20 combined with gemcitabine/nab-paclitaxel in stage 4 PDAC patients showed promising results of adding PEGPH20 to chemotherapy in a subgroup of patients with HA-positive tumor biopsies ${ }^{42}$. Interestingly, preliminary data based on retrospective analysis of HALO202 also suggested that the C3M/PRO-C3 ratio was able to identify a subgroup of patients with better benefit of $\mathrm{PEGPH} 20^{43}$. Together these findings suggest not only prognostic potential of C3M and PRO-C3, but also the ability to predict the likelihood of responding to a given combination of modalities. With the next wave of stroma/ ECM-targeting therapy for PDAC on its way, inclusion of non-invasive biomarkers to identify specific subtypes of PDAC with ongoing desmoplasia is an important step forward ${ }^{44-46}$. However, to include the present biomarkers in clinical trial decision-making, the present study needs to be validated in larger, well characterized clinical cohorts, since the present result is limited by a relatively small sample size with limited available clinical information and is a retrospective biomarker analysis for predicting outcome.

In conclusion, this study shows that collagen remodeling biomarkers measured in pre-treatment serum are highly elevated and associated with OS in PDAC patients, supporting the link between the desmoplastic reaction, tumorigenesis, prognosis, and response to treatment. Of the four biomarkers evaluated, $\mathrm{PRO}-\mathrm{C} 3$, a biomarker of type III collagen formation, appeared to provide the best prognostic value, independent of CA19-9. In summary, these results aid in the understanding of ECM turnover (formation and degradation of different collagens) as an important component in tumor progression and/or response to treatment. If validated in larger clinical cohorts, these collagen biomarkers may have potential as prognostic and/or predictive biomarkers in the PDAC setting.

\section{Data availability}

The datasets used and/or analysed during the current study are available from the corresponding author on reasonable request.

Received: 5 August 2019; Accepted: 8 December 2019;

Published online: 24 December 2019

\section{References}

1. Siegel, R. L., Miller, K. D. \& Jemal, A. Cancer statistics, 2018. CA. Cancer J. Clin. 68, 7-30 (2018).

2. Rahib, L. et al. Projecting cancer incidence and deaths to 2030: the unexpected burden of thyroid, liver, and pancreas cancers in the United States. Cancer Res. 74, 2913-21 (2014).

3. Teague, A., Lim, K.-H. \& Wang-Gillam, A. Advanced pancreatic adenocarcinoma: a review of current treatment strategies and developing therapies. Ther. Adv. Med. Oncol. 7, 68-84 (2015).

4. Adamska, A., Domenichini, A. \& Falasca, M. Pancreatic Ductal Adenocarcinoma: Current and Evolving Therapies. Int. J. Mol. Sci. 18 (2017).

5. Balaban, E. P. et al. Locally advanced, unresectable pancreatic cancer: American society of clinical oncology clinical practice guideline. J. Clin. Oncol. 34, 2654-2667 (2016).

6. Feig, C. et al. The pancreas cancer microenvironment. Clin Cancer Res 18, 4266-4276 (2013).

7. Whatcott, C. J. et al. Desmoplasia in primary tumors and metastatic lesions of pancreatic cancer. Clin. Cancer Res. 21, 3561-3568 (2015).

8. Cannon, A. et al. Desmoplasia in pancreatic ductal adenocarcinoma: insight into pathological function and therapeutic potential. Genes Cancer 9, 78-86 (2018).

9. Rosenberg, A. \& Mahalingam, D. Immunotherapy in pancreatic adenocarcinoma-overcoming barriers to response. J. Gastrointest. Oncol. 9, 143-159(2018).

10. Lu, P., Weaver, V. M. \& Werb, Z. The extracellular matrix: a dynamic niche in cancer progression. J.Cell Biol. 196, 395-406 (2012).

11. Karsdal, M. A. et al. Extracellular matrix remodeling: the common denominator in connective tissue diseases. Possibilities for evaluation and current understanding of the matrix as more than a passive architecture, but a key player in tissue failure. Assay Drug Dev. Technol. 11, 70-92 (2013).

12. Bonnans, C., Chou, J. \& Werb, Z. Remodelling the extracellular matrix in development and disease. Nat. Rev. Mol. Cell Biol. 15, 786-801 (2014).

13. Filipe, E. C., Chitty, J. L. \& Cox, T. R. Charting the unexplored extracellular matrix in cancer. Int. J. Exp. Pathol. 99, 58-76 (2018).

14. Yen, T. W. F. et al. Myofibroblasts are responsible for the desmoplastic reaction surrounding human pancreatic carcinomas. Surgery 131, 129-34 (2002).

15. Apte, M. V. et al. Desmoplastic Reaction in Pancreatic Cancer. Pancreas 29, 179-187 (2004).

16. Kessenbrock, K., Plaks, V. \& Werb, Z. Matrix metalloproteinases: regulators of the tumor microenvironment. Cell 141, 52-67 (2010).

17. Shields, M. A., ngi-Garimella, S., Redig, A. J., Munshi, H. G. \& Dangi-Garimella, S. Biochemical role of the collagen-rich tumour microenvironment in pancreatic cancer progression. Biochem.J. 441, 541-552 (2012).

18. Armstrong, T. et al. Type I Collagen Promotes the Malignant Phenotype of Pancreatic Ductal Adenocarcinoma. Clin. Cancer Res. 10, 7427-7437 (2004).

19. Imamura, T. et al. Quantitative analysis of collagen and collagen subtypes I, III, and V in human pancreatic cancer, tumor-associated chronic pancreatitis, and alcoholic chronic pancreatitis. Pancreas 11, 357-64 (1995).

20. Menke, A. et al. Down-Regulation of E-Cadherin Gene Expression by Collagen Type I and Type III in Pancreatic Cancer Cell Lines. Biochemistry 61, 3508-3517 (2001). 
21. Öhlund, D. et al. Type IV collagen stimulates pancreatic cancer cell proliferation, migration, and inhibits apoptosis through an autocrine loop. BMC Cancer 13, 154 (2013).

22. Gascard, P. \& Tlsty, T. D. Carcinoma-associated fibroblasts: Orchestrating the composition of malignancy. Genes Dev. 30, 1002-1019 (2016).

23. Theocharis, A. D., Skandalis, S. S., Gialeli, C. \& Karamanos, N. K. Extracellular matrix structure. Adv. Drug Deliv. Rev. 97, 4-27 (2016).

24. Kalluri, R. \& Zeisberg, M. Fibroblasts in cancer. Nat.Rev.Cancer 6, 392-401 (2006)

25. Kalluri, R. The biology and function of fibroblasts in cancer. Nat. Rev. Cancer 16, 582-598 (2016).

26. Willumsen, N. et al. Extracellular matrix specific protein fingerprints measured in serum can separate pancreatic cancer patients from healthy controls. BMC Cancer 13, 554 (2013).

27. Roy, A. et al. Phase 3 trial of SMS 201-995 pa LAR (SMS PA LAR) and continuous infusion 5FU in unresectable stage II, III, and IV pancreatic cancer. Proc Am Soc Clin Oncol 17, 257 (1998).

28. Alkhateeb, A. et al. Elevation in multiple serum inflammatory biomarkers predicts survival of pancreatic cancer patients with inoperable disease. J. Gastrointest. Cancer 45, 161-167 (2014).

29. Leeming, D. et al. A novel marker for assessment of liver matrix remodeling: an enzyme-linked immunosorbent assay (ELISA) detecting a MMP generated type I collagen neo-epitope (C1M). Biomarkers 16, 616-628 (2011).

30. Barascuk, N. et al. A novel assay for extracellular matrix remodeling associated with liver fibrosis: An enzyme-linked immunosorbent assay (ELISA) for a MMP-9 proteolytically revealed neo-epitope of type III collagen. Clin. Biochem. 43, 899-904 (2010).

31. Veidal, S. S. et al. Assessment of proteolytic degradation of the basement membrane: a fragment of type IV collagen as a biochemical marker for liver fibrosis. Fibrogenesis. Tissue Repair. 4, 22 (2011).

32. Nielsen, M. J. et al. The neo-epitope specific PRO-C3 ELISA measures true formation of type III collagen associated with liver and muscle parameters. Am. J. Transl. Res. 5, 303-315 (2013).

33. Kehlet, S. N. et al. Age-related collagen turnover of the interstitial matrix and basement membrane: Implications of age- and sexdependent remodeling of the extracellular matrix. PLoS One 13, e0194458 (2018).

34. Willumsen, N. et al. Serum biomarkers reflecting specific tumor tissue remodeling processes are valuable diagnostic tools for lung cancer. Cancer Med. 3, 1136-1145.

35. Bager, C. L. et al. Collagen degradation products measured in serum can separate ovarian and breast cancer patients from healthy controls: A preliminary study. Cancer Biomark. 15, 783-788 (2015).

36. Lipton, A. et al. High turnover of extracellular matrix reflected by specific protein fragments measured in serum is associated with poor outcomes in two metastatic breast cancer cohorts. Int. J. Cancer 143, 3027-3034 (2018).

37. Jensen, C. et al. Non-invasive biomarkers derived from the extracellular matrix associate with response to immune checkpoint blockade (anti- CTLA-4) in metastatic melanoma patients. J. Immunother. Cancer 6, 1-10 (2018).

38. Chen, I. et al. Clinical value of serum hyaluronan and propeptide of type III collagen in patients with pancreatic cancer. Int. J. Cancer (2019).

39. Nissen, N. I., Karsdal, M. \& Willumsen, N. Collagens and Cancer associated fibroblasts in the reactive stroma and its relation to Cancer biology. J. Exp. Clin. Cancer Res. 38, 115 (2019).

40. Provenzano, P. P. et al. Enzymatic Targeting of the Stroma Ablates Physical Barriers to Treatment of Pancreatic Ductal Adenocarcinoma. Cancer Cell 21, 418-429 (2012).

41. Wong, K. M., Horton, K. J., Coveler, A. L., Hingorani, S. R. \& Harris, W. P. Targeting the Tumor Stroma: the Biology and Clinical Development of Pegylated Recombinant Human Hyaluronidase (PEGPH20). Curr. Oncol. Rep. 19 (2017).

42. Hingorani, S. R. et al. HALO 202: Randomized phase II Study of PEGPH20 Plus Nab-Paclitaxel/Gemcitabine Versus Nab-Paclitaxel/ Gemcitabine in Patients With Untreated, Metastatic Pancreatic Ductal Adenocarcinoma. J. Clin. Oncol. 36, 359-366 (2018).

43. Wang, S. et al. Extracellular matrix (ECM) circulating peptide biomarkers as potential predictors of survival in patients (pts) with untreated metastatic pancreatic ductal adenocarcinoma (mPDA) receiving pegvorhyaluronidase alfa (PEGPH20), nab-paclitaxel (A), and gemcitabine (G). J. Clin. Oncol. 36, 12030 (2018).

44. Huang, H. \& Brekken, R. A. The next wave of stroma-targeting therapy in pancreatic cancer. Cancer Res. 79, 328-330 (2019).

45. Elahi-Gedwillo, K. Y., Carlson, M., Zettervall, J. \& Provenzano, P. P. Antifibrotic therapy disrupts stromal barriers and modulates the immune landscape in pancreatic ductal adenocarcinoma. Cancer Res. 79, 372-386 (2019).

46. Vennin, C. et al. Reshaping the Tumor Stroma for Treatment of Pancreatic Cancer. Gastroenterology 154, 820-838 (2018).

\section{Acknowledgements}

We acknowledge the Danish Research Foundation for supporting the biomarker measurements in this study.

\section{Author contributions}

N.W., S.M.A., K.L., M.A.K. and A.L. did the conceptualization; N.W. did the biomarker measurements and wrote the original first draft; All authors took part in analysis and interpretation of data and read and approved the final manuscript.

\section{Competing interests}

Nicholas Willumsen and Morten A. Karsdal have declared financial competing interest from employment at Nordic Bioscience involved in biomarker discovery and development. All remaining authors have declared no financial conflicts of interest. None of the authors have non-financial competing interest.

\section{Additional information}

Supplementary information is available for this paper at https://doi.org/10.1038/s41598-019-56268-3.

Correspondence and requests for materials should be addressed to N.W.

Reprints and permissions information is available at www.nature.com/reprints.

Publisher's note Springer Nature remains neutral with regard to jurisdictional claims in published maps and institutional affiliations. 
(c) (i) Open Access This article is licensed under a Creative Commons Attribution 4.0 International License, which permits use, sharing, adaptation, distribution and reproduction in any medium or format, as long as you give appropriate credit to the original author(s) and the source, provide a link to the Creative Commons license, and indicate if changes were made. The images or other third party material in this article are included in the article's Creative Commons license, unless indicated otherwise in a credit line to the material. If material is not included in the article's Creative Commons license and your intended use is not permitted by statutory regulation or exceeds the permitted use, you will need to obtain permission directly from the copyright holder. To view a copy of this license, visit http://creativecommons.org/licenses/by/4.0/.

(C) The Author(s) 2019 